\title{
Metastatic adnexal cancer in a man
}

\author{
Rashmi Advania , Manhal Izzy ${ }^{a}$, Ashwin Akki ${ }^{b}$, Calley Levine ${ }^{a}$, Nicole Panarellib, Lawrence J. Brandt ${ }^{a}$ \\ Montefiore Medical Center, Albert Einstein College of Medicine, Bronx, NY, USA
}

\begin{abstract}
Primary cutaneous adnexal neoplasms are mostly benign in nature; however, there have been reports of malignant adnexal tumors with distant metastasis to lymph nodes. Adnexal cutaneous malignancy with metastasis to the gastrointestinal tract has never been reported. Here, we present a rare case of a man with primary adnexal cutaneous adenocarcinoma who presented with symptomatic anemia secondary to occult gastrointestinal bleeding, found to be from gastrointestinal metastasis of the adnexal malignancy.
\end{abstract}

Keywords Primary cutaneous adnexal neoplasms, gastrointestinal malignancy, skin tumor

Ann Gastroenterol 2017; 30 (4): 1-2

\section{Introduction}

Primary cutaneous adnexal neoplasms (PCANs) are a group of tumors that involve various lines of cellular differentiation, originating from sebaceous glands, apocrine glands, eccrine glands, and/or hair follicles [1-3]. Although their exact prevalence is unknown, these lesions are rare compared with other skin tumors [4]. Most of these neoplasms are benign; however, they may present as malignant lesions with distant metastasis, usually to lymph nodes [1]. Here, we present a rare case of a man with a poorly differentiated primary adnexal cutaneous adenocarcinoma metastasized to the gastrointestinal (GI) tract.

\section{Case report}

A 74-year-old man presented with a soft, cystic, and enlarging mass of the right superior temporal region that measured $7.4 \times 4.4 \mathrm{~cm}$. Histopathology was consistent

${ }^{a}$ Division of Gastroenterology and Liver Diseases (Rashmi Advani,

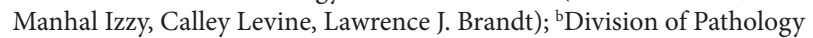
(Ashwin Akki, Nicole Panarelli), Montefiore Medical Center, Albert Einstein College of Medicine, Bronx, NY

Conflict of Interest: None

Correspondence to: Manhal Izzy, MD, Montefiore Medical Center, Albert Einstein College of Medicine, Division of Gastroenterology, 111 East $210^{\text {th }}$ Street, Bronx, NY 10467, USA, Tel.: +1 7189204154 , e-mail:mizzy@montefiore.org

Received 04 April 2017; accepted 27 April 2017; published online 12 May 2017

DOI: https://doi.org/10.20524/aog.2017.0156 with carcinoma that extended into the perineural and lymphovascular spaces, and showed immunoreactivity to cytokeratin CK5, CK6, CK7, and anti-epithelial cell adhesion molecule BerEp4 (Fig. 1A, 1B). No reactivity was detected to CK20, chromogranin, or synaptophysin. Consequently, the diagnosis of PCAN was made. Radiolabeled [18F]-2-fluoro-2deoxy-D-glucose uptake was noted in the colon on a positron emission tomography (PET) scan; however, a subsequent colonoscopy was unremarkable. No other abnormalities were noted on the PET scan. A year later, while on radiation therapy, the patient was diagnosed with bone and lymph node metastasis. During the second year of the disease course, he presented with flank pain, at which point a computed tomography scan of the abdomen/pelvis showed nodular thickening of the gastric wall. Two months later, the patient developed symptomatic anemia and occult GI bleeding. Upper endoscopy was performed and revealed multiple raised umbilicated nodules with central ulceration, ranging from $0.7-3 \mathrm{~cm}$, throughout the gastric body (Fig. 2A, 2B) and the second portion of the duodenum (Fig. 2C). A biopsy specimen yielded a cellular morphology and immunophenotype identical to the prior scalp biopsy (Fig. 3A, 3B). A diagnosis of metastatic PCAN was made.

\section{Discussion}

PCANs originate from one of the four primary adnexal structures in human skin: hair follicles, apocrine glands, eccrine glands, or sebaceous glands [1-3]. Most PCANs are benign lesions. Malignant adnexal neoplasms resemble their benign counterparts, but are characterized by a tendency to grow rapidly and ulcerate [5]. Metastasis to the GI tract from these skin tumors has never been reported. Conversely, GI malignancies can mimic PCAN by metastasizing to the skin, which occurs in less than $10 \%$ of liver or colon cancers and 


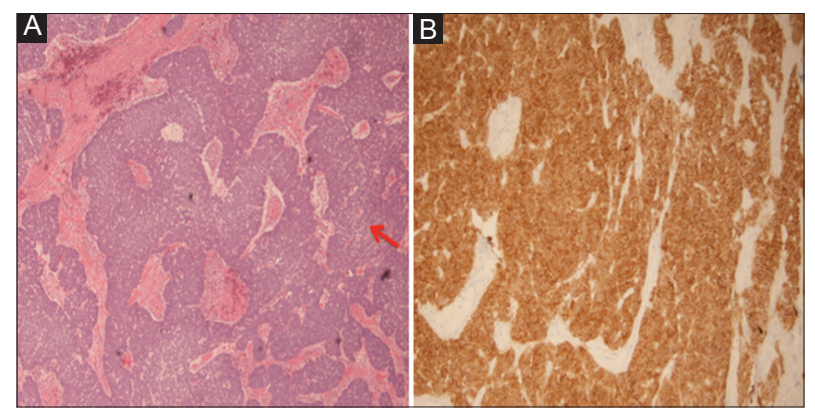

Figure 1 (A) Hematoxylin \& eosin stain of the scalp lesion, showing nests and sheets of tumor cells (arrow) in the dermis and subcutaneous tissue. The tumor cells are round to irregular with enlarged nuclei, coarse chromatin, minimal cytoplasm and frequent mitoses. (B) Tumor cells from the scalp lesion, showing diffuse and strong membranous immunoreactivity for BerEp4

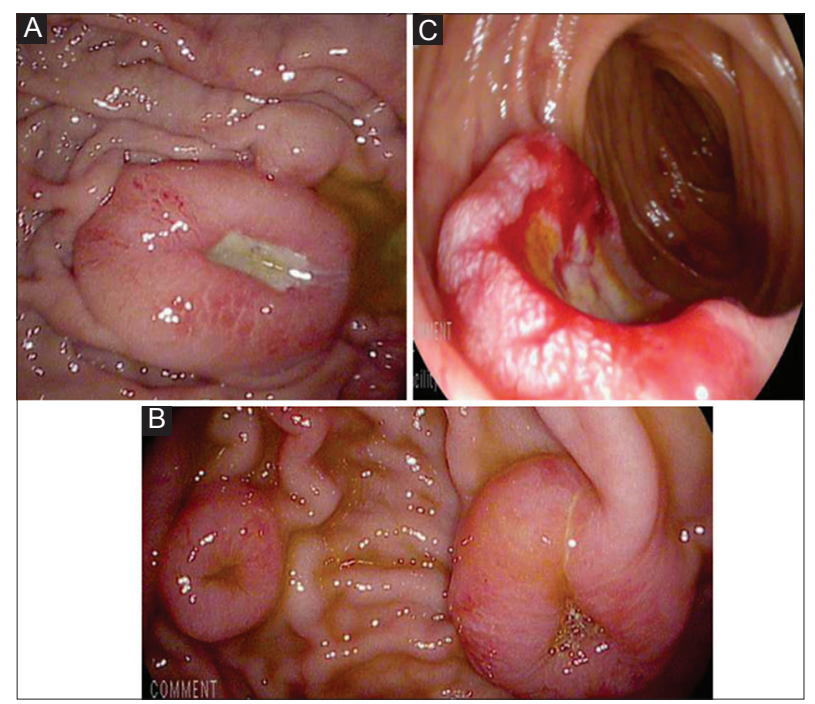

Figure 2 (A) Endoscopic image of a centrally umbilicated nodule in the gastric body. (B) Endoscopic image of two centrally umbilicated nodules of varying diameter in the gastric body. (C) Endoscopic image of an ulcerated, nodular lesion in the duodenum

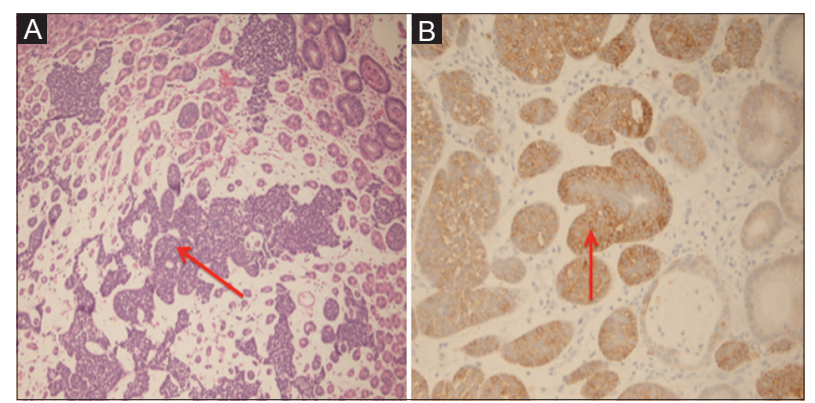

Figure 3 (A) Hematoxylin \& eosin stain, showing gastric oxyntic mucosa undermined by nests and sheets of polygonal tumor cells (arrow) with enlarged hyperchromatic nuclei, coarse chromatin, and minimal cytoplasm, similar to that in the scalp lesion. (B) Tumor cells in the gastric lesion show diffuse and strong membranous immunoreactivity for BerEp4 (arrow), while the background oxyntic mucosa is immunonegative even fewer cases of gallbladder and gastric cancer [6]. Because of the histologic overlap between cutaneous metastasis from GI sites and GI metastasis from malignant PCAN, immunohistochemical staining usually guides the definitive diagnosis [1].

The CK5\&6 positive immunohistochemical stain of the scalp in this patient is highly specific and sensitive for the diagnosis of PCAN $[2,7,8]$. CK7 positivity is additionally consistent with the diagnosis of PCAN. Lack of CK7 usually indicates a metastatic tumor from the GI tract [1]. Lastly, negative staining with CK20, CDX2, and chromogranin with synaptophysin further supports the diagnosis of metastatic PCAN; their positivity would have reflected a primary GI malignancy or a neuroendocrine tumor, respectively $[2,3,5]$.

Data on the treatment of metastatic adnexal tumors are very scarce and involve chemotherapeutic agents such as platinum-based agents, taxanes, anthracyclines, adriamycin, and bleomycin [9]. In contrast, GI malignancies metastasized to the skin are typically treated with a combination of chemotherapeutic agents including platinum agents, taxanes, irinotecan and/or trastuzumab (for tumors expressing HER-2) [10].

In summary, we present the first case of PCAN metastasis to the upper GI tract involving the stomach and duodenum. It is important to differentiate this rare lesion from cutaneous metastases from a GI malignancy, in order to allow appropriate treatment and potentially improve prognosis.

\section{References}

1. Danialan R, Mutyambizi K, Aung P, Prieto VG, Ivan D. Challenges in the diagnosis of cutaneous adnexal tumours. J Clin Pathol 2015;68:992-1002.

2. Crowson AN, Magro CM, Mihm MC. Malignant adnexal neoplasms. Mod Pathol 2006;19(Suppl 2):S93-S126.

3. Alsaad KO, Obaidat NA, Ghazarian D. Skin adnexal neoplasmspart 1: an approach to tumours of the pilosebaceous unit. J Clin Pathol 2007;60:129-144.

4. Martinez SR, Barr KL, Canter RJ. Rare tumors through the looking glass: an examination of malignant cutaneous adnexal tumors. Arch Dermatol 2011;147:1058-1062.

5. Obaidat NA, Alsaad KO, Ghazarian D. Skin adnexal neoplasmspart 2: an approach to tumours of cutaneous sweat glands. J Clin Pathol 2007;60:145-159.

6. Sariya D, Ruth K, Adams-McDonnell R, et al. Clinicopathologic correlation of cutaneous metastases: experience from a cancer center. Arch Dermatol 2007;143:613-620.

7. Qureshi HS, Ormsby AH, Lee MW, Zarbo RJ, Ma CK. The diagnostic utility of p63, CK5/6, CK 7, and CK 20 in distinguishing primary cutaneous adnexal neoplasms from metastatic carcinomas. J Cutan Pathol 2004;31:145-152.

8. Plumb SJ, Argenyi ZB, Stone MS, De Young BR. Cytokeratin 5/6 immunostaining in cutaneous adnexal neoplasms and metastatic adenocarcinoma. Am J Dermatopathol 2004;26:447-451.

9. De Iuliis F, Amoroso L, Taglieri L, et al. Chemotherapy of rare skin adnexal tumors: a review of literature. Anticancer Res 2014;34:5263-5268.

10. Japanese Gastric Cancer Association. Japanese gastric cancer treatment guidelines 2014 (ver. 4). Gastric Cancer 2017;20:1-19. 